\title{
Using a PANS Simulation Approach for the Transient Flow around the Japan Bulk Carrier
}

\author{
R.E. Bensow ${ }^{1}$, and M. van den Boogaard ${ }^{2}$ \\ ( ${ }^{1}$ Chalmers University of Technology, Sweden, ${ }^{2}$ Delft University of Technology, \\ The Netherlands)
}

\begin{abstract}
In this study, a PANS, Partially averaged Navier-Stokes, modelling approach developed based on the $k-\omega$ turbulence model has been applied to the flow around the Japan Bulk Carrier. Three different meshes has been employed for a fixed physical resolution for the PANS modelling. The results are encouraging, with small scale flow dynamics being allowed to develop on reasonably small mesh sizes, but more studies are required before reliable predictive simulations can be performed.
\end{abstract}

\section{INTRODUCTION}

Steady resistance and wake simulations in ship hydrodynamics are currently being performed with relatively high reliability using RANS simulation approaches. There are however a number of cases where RANS is not sufficient and a scale resolving transient simulation approach is needed, it could be that details of the transient behaviour is part of the study, e.g. relating to noise and vibration, or that the flow is separating, or close to, and RANS modelling is not sufficient to capture the governing flow physics, e.g. at larger yaw angles. As wall-resolved LES is very computationally expensive, with an unfavourable scaling towards full scale $R e$ numbers, the attention has rather turned towards hybrid RANS/LES approaches or wall modelled LES.

The Partially Averaged Navier-Stokes approach was suggested by Girimaji (2005) as a turbulence modelling approach where the physical resolution can be chosen seamlessly between RANS (fully modelled) and DNS (fully resolved). It is often called a bridging hybrid method, in contrast to the more common hybrid RANS/LES methods, such as DES (Spalart et al., 1997), which are based on a zonal geometrical decomposition between the modelling approaches. PANS has proven itself in a number of applications, e.g. (Frendi et al. 2007. Krajnovic et al. 2012, Pereira, 2018), however primarily for bluff body flows where turbulence develops in shear layers. More recently, studies have been extended to boundary layer flows by Razi (2017), where the properties for using PANS for a channel flow were investigated.

To our knowledge, PANS has not previously been applied to flows characterised by a long developing boundary layer with mild separation, as is the situation for many ship hulls. There is thus no precedence regarding requirements on turbulence modelling and mesh resolution, and this paper is a first attempt at clarifying these questions and the possible potential for using PANS in ship hydrodynamics.

The flow chosen for this study is the Japan Bulk Carrier, used at the Tokyo 2015 Workshop on Numerical Ship Hydrodynamics. As discussed by Visonneau et al. (2016), the analysis of the results of the participants of the workshop indicated that this vessel had a stern flow field more difficult to compute with the usual RANS approaches, due to the high block coefficient. In particular the structure and turbulence content of the aft bilge vortex showed large discrepancies between RANS predictions and measurements. Simulations performed using DES approaches, by Abbas and Kornev (2015) for the workshop and later by Visonneau et al. (2016), were however much closer to the experiments indicating a flow development that RANS is not able to capture.

The presented results, using PANS, indicate somewhat mixed performance. On the grids tested, the global drag force is somewhat under predicted, indicating that wall resolution is not sufficient for the corresponding physical resolution chosen in the PANS. Moreover, the average flow field does not show a satisfactory development of the aft bilges, although not significantly worse than most RANS results presented at the workshop. However, medium scale flow structures develop already on relatively coarse grids, indicating a promising behaviour.

\section{PANS MODELLING}

In PANS, the resolved and modelled scales are decomposed via an arbitrary filter. The amount of 
modelling is determined via the unresolved to total turbulent kinetic energy and dissipation, respectively,

$$
f_{k}=\frac{k_{u}}{k}, \quad f_{\varepsilon}=\frac{\varepsilon_{u}}{\varepsilon} .
$$

Most often, the models are developed based on established RANS models, which have proven to give reasonable results and robust behaviour in many cases. Moreover, in the limit of $f_{k}=f_{\varepsilon}=1$, a RANS behaviour is expected.

In the current work, the PANS formulation developed by Lakshmipathy (2009) has been adapted in OpenFOAM by modifying the implementation of the $k-$ $\omega$-model of Wilcox (1998). The model equations for the unresolved kinetic turbulent energy, $k_{u}$, and unresolved specific dissipation rate, $\omega_{u}$, are,

$$
\begin{aligned}
\frac{\partial k_{u}}{\partial t}+U_{j} \frac{\partial k_{u}}{\partial x_{j}}= & P_{u}-\beta^{*} k_{u} \omega_{u}+ \\
& +\frac{\partial}{\partial x_{j}}\left(v+\frac{v_{u}}{\sigma_{k u}} \frac{\partial k_{u}}{\partial x_{j}}\right), \\
\frac{\partial \omega_{u}}{\partial t}+U_{j} \frac{\partial \omega_{u}}{\partial x_{j}}= & \gamma \frac{P_{u} \omega_{u}}{k_{u}}-\beta^{\prime} \omega_{u}^{2}+ \\
& +\frac{\partial}{\partial x_{j}}\left(v+\frac{v_{u}}{\sigma_{\omega u}} \frac{\partial \omega_{u}}{\partial x_{j}}\right),
\end{aligned}
$$

where, $\beta^{\prime}=\gamma \beta^{*}-\frac{\gamma \beta^{*}}{f_{\omega}}+\frac{\beta}{f_{\omega}}$, and $\quad \sigma_{k u}=\sigma_{k} \frac{f_{k}}{f_{\omega}}, \quad \sigma_{\omega u}=$ $\sigma_{\omega} \frac{f_{k}}{f_{\omega}}$. The unresolved turbulent viscosity is computed as $v_{u}=k_{u} / \omega_{u}$. As high Re number flow is considered for this work, $f_{\varepsilon}=1$ and simulations are run for different (spatially constant) values of $f_{k}$.

\section{COMPUTATIONAL CONFIGURATION}

The simulations are performed in model scale for a double body model, as the $F n$ number is reasonably low and the waves are not expected to significantly influence the local flow leading into the propeller disc. Drag forces are however affected, as there will here be no contribution from wave resistance. It is however considered an acceptable simplification. The full hull is however simulated, as the transient flow is not symmetric around the centre line. The outer domain extends $2 L_{P P}$ upstream of the bow, $3 L_{P P}$ downstream the stern, $1.5 L_{P P}$ below the hull and $2 L_{P P}$ on each side.

To get an impression of the resolution requirements of PANS, three different meshes have been used, see Table 1 and Figure 1 . The starting point was a mesh developed for RANS and shown to be satisfactory in Andersson et al. (2015); this mesh consisted of about 3 million cells for half hull in double body configuration. As the main objective for using PANS would be to allow small scale structures to develop, a refinement box was added in the stern, giving the first PANS mesh, denoted M2. For the second mesh, M3, the original RANS mesh was refined around the complete hull resulting in similar cell sizes in the aft as for M2, but with finer mesh in the hull boundary layer. The final mesh was again one complete refinement on the M3 mesh.

On the coarse mesh, three different values of $f_{k}$ were simulated, $f_{k}=1.0,0.5,0.25$, while on the finer meshes only the higher physical resolution, $f_{k}=0.25$, has been considered. Note that $f_{k}=1.0$ corresponds to RANS modelling.

The simulations were allowed to run to a developed flow before sampling statistics was initiated. Within the time frame for this study, the simulation on the coarsest M2 mesh have only run for about 2.5 hull pass through times for gathering statistices, so it's clear that statistical convergence has not been achieved. For the finer meshes M3 and M4, at least 4 hull pass times have been used for statistics. Time step was chosen to achieve a $C o$ number below one in the entire flow domain.

The flow is simulated using the open source software package OpenFOAM 1 , which provides an object-oriented library, based on the finite-volume method, specifically designed for CFD; see Weller et al. (1997) for a description of the structure of this software design.

The discretization of the governing flow equations relies on storage of the unknown flow variables in the cell-centre positions in the computational grid. The algorithm supports arbitrary polyhedral cells and the grid is treated as unstructured. The approximations involved are of second-order accuracy, except for flux limiting for the convective term, which reduces locally the formal order of accuracy near sharp gradients. The momentum equation is treated in a segregated manner using the PISO algorithm. The simulations are time resolved and a second order backward differencing scheme is used for the time advancement of the components of the momentum equation.

Table 1: Computational meshes

\begin{tabular}{llll}
\hline & No cells & $\Delta x^{+}$ & $\Delta y^{+}$ \\
\hline M2 & $14,742,455$ & 175 & 0.45 \\
M3 & $19,140,493$ & 130 & 0.45 \\
M4 & $31,701,633$ & 100 & 0.45 \\
\hline
\end{tabular}

$1_{\text {WWW }}$ openfoam. com 


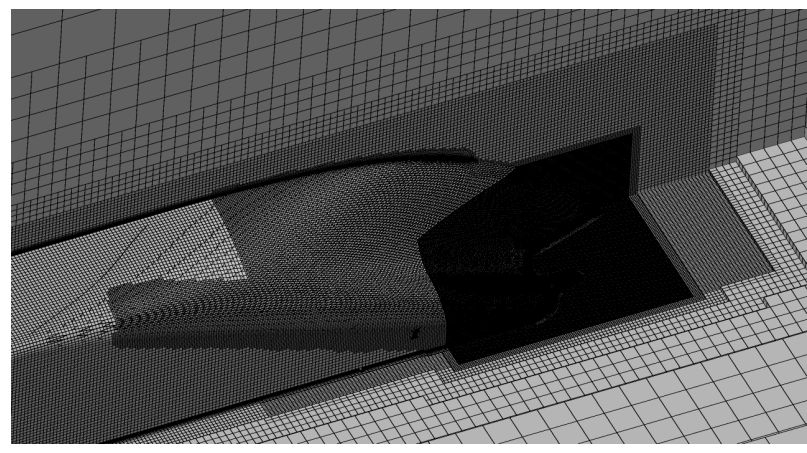

(a) M2

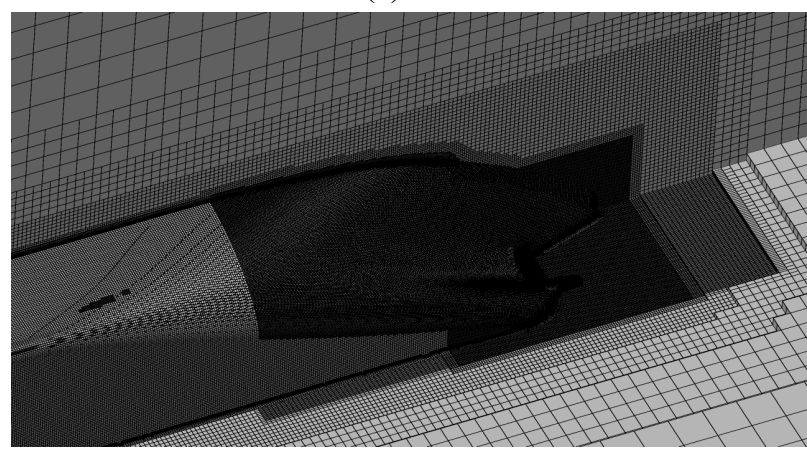

(b) M3

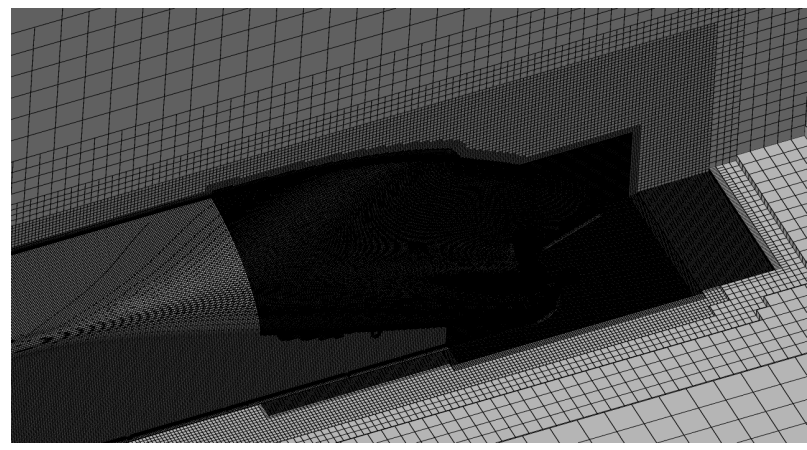

(c) M4

Figure 1: The three meshes used for the PANS simulations.

\section{RESULTS}

Here, first the global drag prediction is presented before making a more detailed analysis of PANS performance for local flow prediction. Post-processing is performed in accordance with the instructions given to participants of the Tokyo 2015 workshop.

\section{Force prediction}

The predicted drag is presented in Table 2 with comparison to the towing test results by NMRI presented at the Tokyo workshop as well as the RANS prediction of ECN and the hybrid RANS/LES prediction of the University of Rostock, as example results from the workshop. Note that these three results are with the free free surface.

We note that the simulation with $f_{k}=1.0$ over predicts the viscous drag, if compared with what the ITTC-57 would give. The over prediction is relatively small and in line with other OpenFOAM predictions from the workshop. The drag of the PANS $f_{k}=0.25$ on the M2 mesh significantly under predicts the viscous drag. The natural interpretation is that resolution is not sufficient to allow representative boundary layer structures consistent with the reduction of turbulent viscosity imposed by $f_{k}=0.25$. The situation does not seem to improved when refining the grid. A potential issue to be further investigated, is how the turbulent structures in the boundary layer are initiated, as the experimental model is tripped while the simulation is not. The under prediction might thus be related to a lack of transition/trip model in the simulation model.

The pressure drag is more or less similarly predicted in all simulations. In contrast to the hybrid RANS/LES results of Abbas and Kornev (2015), the more dynamic flow does not lead to increased pressure drag. The 'more laminar' and thinner boundary layer predicted by PANS here could be expected to be responsible for this under prediction as well.

Table 2: Model ship resistance (towed)

\begin{tabular}{|c|c|c|c|}
\hline & \multicolumn{3}{|c|}{ Free surface } \\
\hline & $R_{V}$ & $R_{P}$ & $R_{T}$ \\
\hline $\mathrm{EFD}^{2}$ & - & - & 35.9 \\
\hline $\mathrm{ECN}^{2}$ & 25.9 & 9.3 & 35.2 \\
\hline \multirow[t]{3}{*}{ U Rostock (HRLES) $^{2}$} & 21.8 & 12.5 & 34.2 \\
\hline & \multicolumn{3}{|c|}{ Double body } \\
\hline & $R_{V}$ & $R_{P}$ & $R_{T}$ \\
\hline ITTC-57 & 26.4 & - & - \\
\hline M2 PANS $f_{k}=1.0$ & 28.1 & 7.1 & 35.2 \\
\hline M2 PANS $f_{k}=0.5$ & 26.1 & 7.8 & 33.9 \\
\hline M2 PANS $f_{k}=0.25$ & 21.0 & 8.5 & 29.5 \\
\hline M3 PANS $f_{k}=0.25$ & 20.7 & 8.9 & 29.6 \\
\hline M4 PANS $f_{k}=0.25$ & 20.7 & 8.8 & 29.5 \\
\hline
\end{tabular}

\section{Flow Results}

The flow around the hull are presented in three ways, focused on the aft ship. Experimental data is

\footnotetext{
${ }^{2}$ Data from the Tokyo Workshop in Numerical Ship Hydrodynamics, 2015
} 
available at two planes in the aft of the ship, the S2 plane somewhat upstream the propeller plane, and S4 close to the propeller plane, see Figure 2. At these planes, normalised axial velocity is presented as contour plots, as well as along two lines through the main bilge vortex, one vertical and one horisontal. The centre of the vortex is determined as the point of maximum axial vorticity of the mean flow in the plane; below we only include results from S4 for this transversal vortex analysis. Flow structures are visualised by iso-surfaces of the normalised Q function, with $Q^{*}=Q \cdot L_{P P}^{2} / U_{\infty}^{2}$, coloured by normalised helicity, $U \cdot \Omega /(\|U\|\|\Omega\|)$.

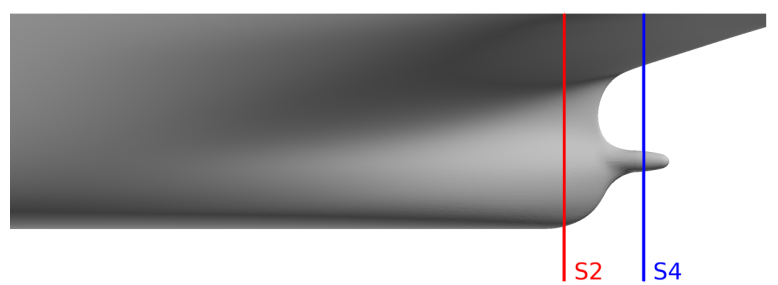

Figure 2: Location of the two evaluation planes.

In Figures 3 and 4 , the predicted axial velocity is compared with the experimental data at the two planes; results are shown for PANS $f_{k}=0.25$ for the three meshes. The results are fairly consistent between the meshes, with some discrepancy believed to be related to lack of statistical convergence on the coarse meshe. The predicted bilge vortex is weaker and somewhat off in location, indicating similar impression as most RANS results for this case.

Isosurfaces of $Q^{*}$ are shown in Figure 5 for three different values of $f_{k}$ on $\mathrm{M} 2$ and in Figure 6 for $f_{k}=0.25$ on M2-M4. In Figure 5a, a RANS like behaviour is predicted, as expected, and as the physical resolution is increased (by decreasing $f_{k}$ ), more transient structures are appearing. On the other hand, keeping the physical resolution constant but increasing the mesh resolution, Figure 6, results in surprisingly similar snapshots on the three meshes. This indicates that the physical and numerical resolution indeed are separated, and that it, in princple, should be possible to achieve a mesh independent solution for every fixed physical resolution; this is in contrast to zonal hybrid RANS/LES and LES approaches where modelling is coupled to cell size.

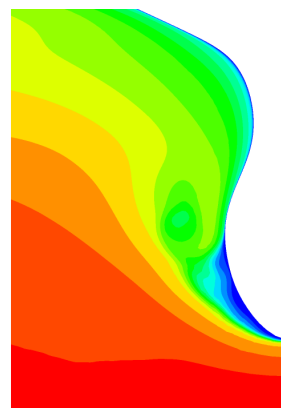

(a) M2

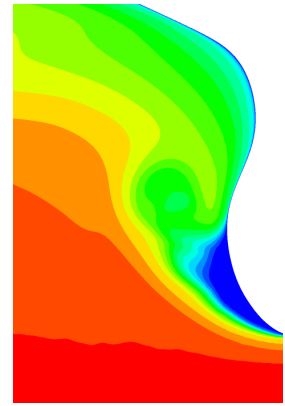

(c) M4

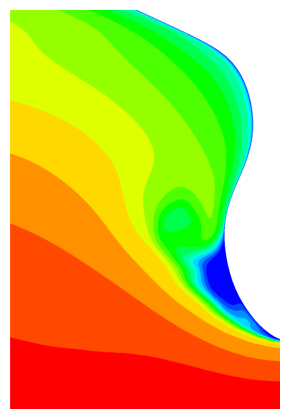

(b) M3

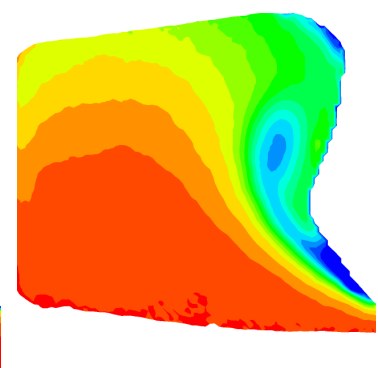

(d) EFD
Figure 3: Axial velocity at S2.

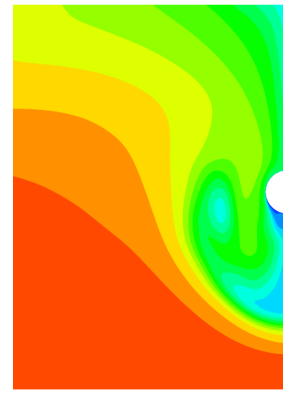

(a) M2

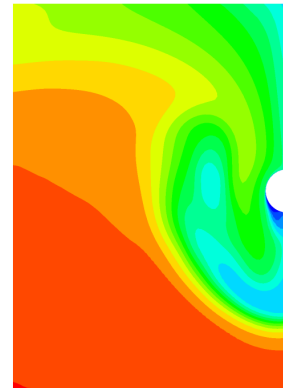

(c) M4

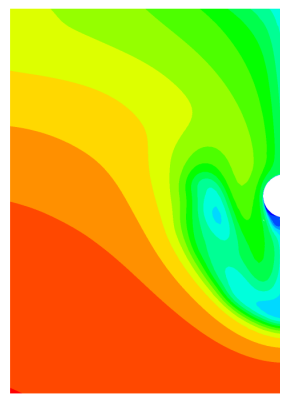

(b) M3

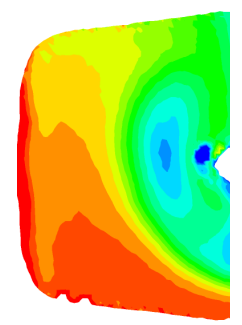

(d) EFD
Figure 4: Axial velocity at S4. 


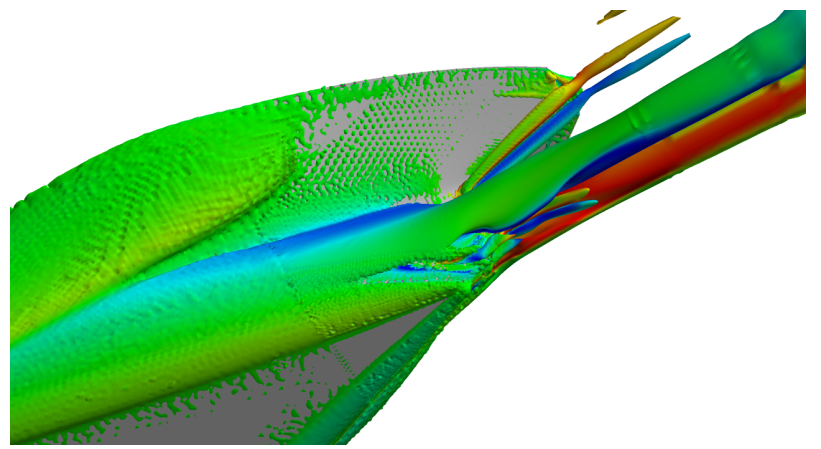

(a) $\mathrm{M} 2, f_{k}=1.0$

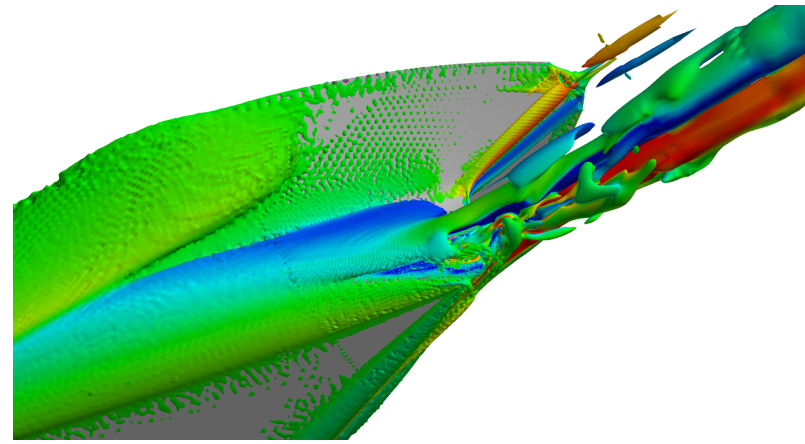

(b) $\mathrm{M} 2, f_{k}=0.5$

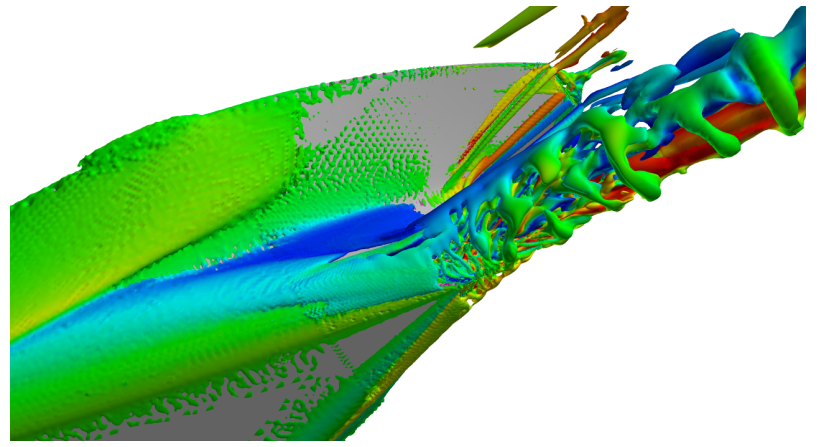

(c) $\mathrm{M} 2, f_{k}=0.25$

Figure 5: Visualisation of the flow structures in the stern using the Q-criterion.

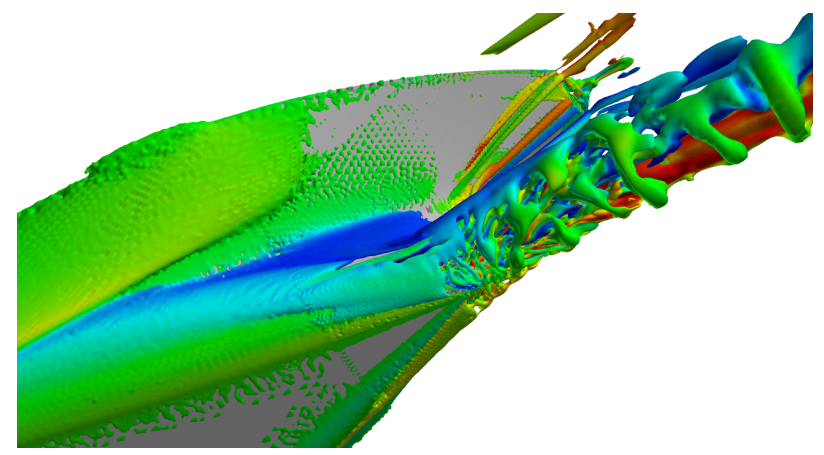

(a) $\mathrm{M} 2$

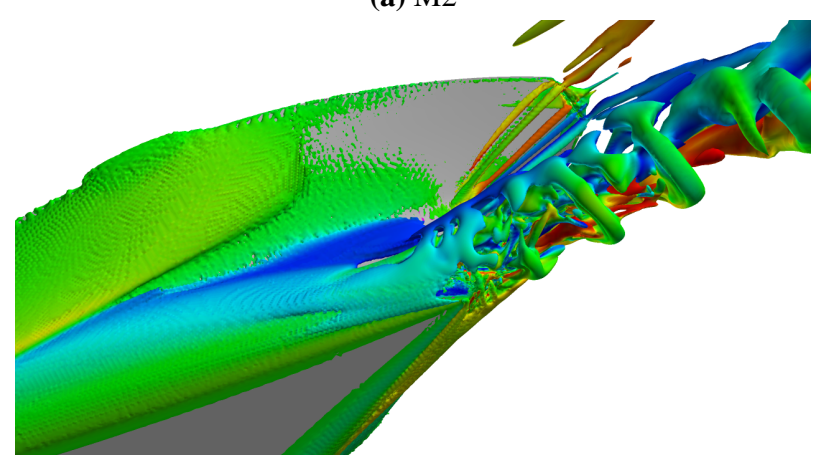

(b) M3

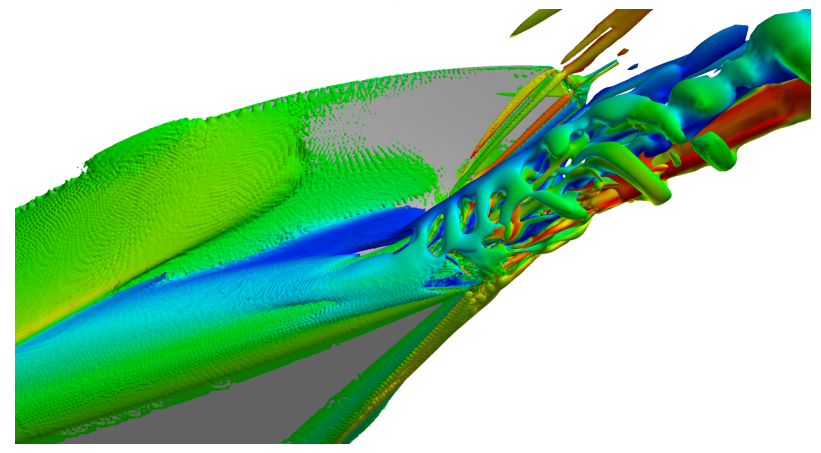

(c) M4

Figure 6: Visualisation of the flow structures in the stern using the Q-criterion. 
Turning to the transversal vortex analysis, for the axial velocity in Figure 7 and the total turbulent kinetic energy, TKE, in Figure 8, one can get a more detailed insight in the predictions. Results are presented for PANS $f_{k}=0.25$ on the three meshes as well as a RANS prediction. The other PANS results are not converged, and, as noted above, the statistics on the caorse grids are not sampled sufficiently long.

It is here clear that the predicted vortex using PANS is narrower than the measured one, while the RANS here yields a decent prediction. For the velocity, Figure 7, there is no clear convergence trend and the results are qualitatively the same on all meshes. For the prediction of turbulence, Figure 8 , it is clear that the PANS results include significantly more dynamics than RANS, and even over predict the unsteadiness compared with the experimental measurements. It is clear that the predictions on M2 give the lowest values of TKE, due to the lack of sufficient sampling time.

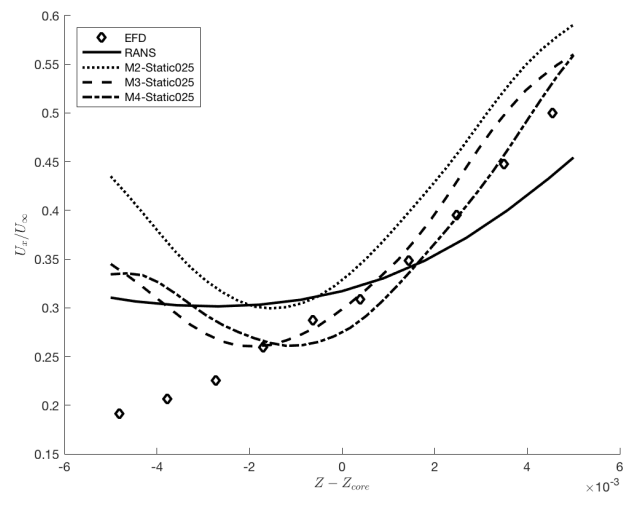

(a)

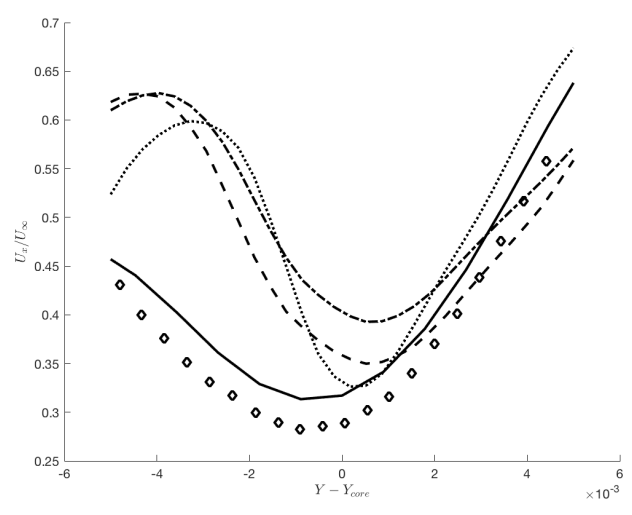

(b)

Figure 7: Axial velocity at S4.

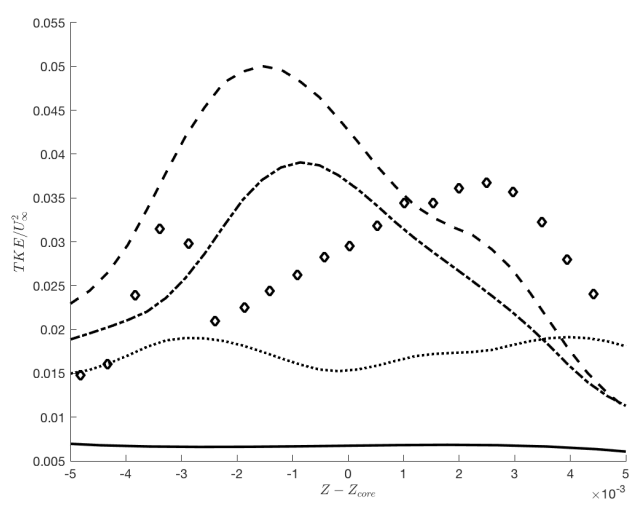

(a)

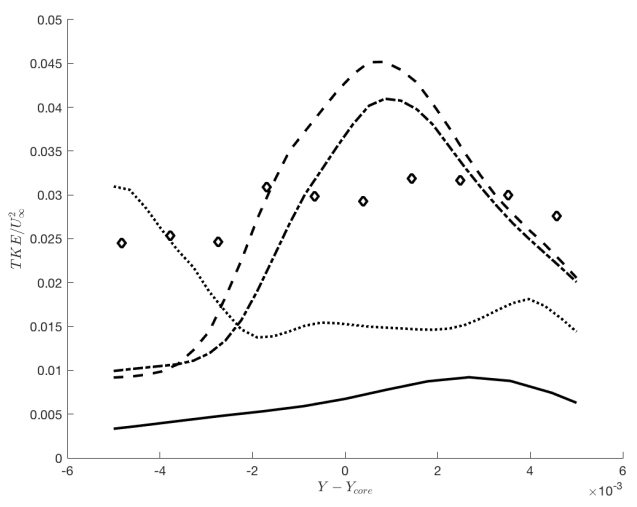

(b)

Figure 8: TKE at S4.
Ideally, a PANS with a fixed $f_{k}$ should display a corresponding reduction in predicted turbulent/unresolved viscosity compared with a RANS. This comparison is tricky to perform, as the RANS predicted flow is steady while the PANS results in a transient flow. To at least indicate the behaviour, the mean turbulent viscosity ratio is shown for the $\mathrm{S} 4$ plane in Figure 9 for different $f_{k}$. One can note that in the vicinity of the bilge vortex, the turbulent viscosity is greatly reduced with a larger reduction for the smaller $f_{k}$, allowing the smaller scale structures to develop, but that outside the vortex turbulent viscosity is increased. This is most probably related to the increase in total turbulent kinetic energy in this region. 


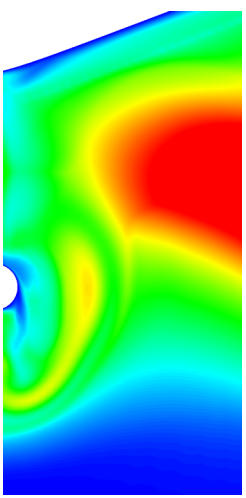

(a) $f_{k}=1.0$

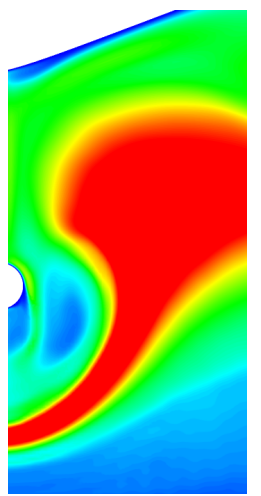

(b) $f_{k}=0.5$

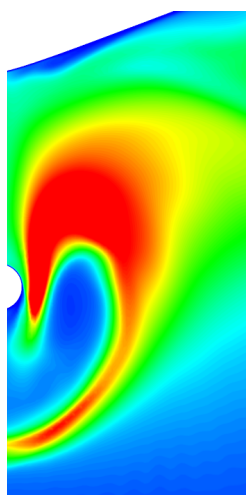

(c) $f_{k}=0.25$

Figure 9: Turbulent viscosity ratio, $v_{u} / v$, at $\mathrm{S} 4$.

\section{CONCLUDING REMARKS}

Simulations of the transient flow around the JBC has been performed using a PANS modelling approach. PANS is attractive due to its potential to deliver resolution by demand, allowing one to use as much modelling as the computational mesh permits; this is in contrast to zonal hybrid RANS/LES or LES where the flow determines the resolution requirements. The approach has proven useful for bluff body flows, but its application for the current type of flow has not been previously investigated. The results are promising but not fully convincing, and further studies are needed to determine the set-up needed to achieve reliable results.

The most important question relates to what is the appropriate $f_{k}$ on a given grid, or alternatively what is the required cell size for a given $f_{k}$. The chosen $f_{k}=0.25$ was inspired by the work of Pereira (2018), and the results here regarding the physical resolution, are consistent with those studies. It would however be interesting to run both smaller and larger $f_{k}$ to see how the results will change, in particular regarding the predicted TKE. Force prediction indicate that the viscous drag is under predicted also on the finest mesh used here, which is quite well resolved in terms of wall units. A trip model of some kind might be necessary to initiate the turbulent structures in the boundary layer.

\section{ACKNOWLEDGEMENTS}

The authors are grateful for the support in mesh generation by Mr. Muye Ge. The simulations were performed on resources at C3SE, Chalmers Centre for Computational Science and Engineering, and NSC, National Supercomputer Centre in Sweden, provided by the Swedish National Infrastructure for Computing (SNIC).

\section{REFERENCES}

Abbas, N. and Kornev, N., "Computations of the Japan Bulk Carrier Using URANS and URANS/LES Methods Implemented into Openfoam Toolkit," Workshop on Numerical Ship Hydrodynamics, Tokyo, 2015.

Andersson, J., Hyensjö, M., Eslamdoost, A., and Bensow, R. E., "CFD Simulations of the Japan Bulk Carrier Test Case," 15th Numerical Towing Tank Symposium, 2015.

Frendi, A., Tosh, A., and Girimaji, S., "Flow past a backward-facing step: Comparison of PANS, des and URANS results with experiments," International Journal of Computational Methods in Engineering Science and Mechanics, 8(1):19-32, 2007 doi: 10.1080/ 15502280601006207.

Girimaji, S., "Partially-Averaged Navier-Stokes Model for Turbulence: A Reynolds-Averaged Navier-Stokes to Direct Numerical Simulation Bridging Method,' J. Appl.Mech., 73(3):413-421, 2005.

Krajnovic, S., Larusson, R., and Basara, B., "Superiority of PANS compared to LES in predicting a rudimentary landing gear flow with affordable meshes," International journal of Heat Fluid Flow, 37:109-122, 2012.

Lakshmipathy, S., "Partially Averaged Navier-Stokes Method for Turbulence," PhD thesis, Texas A\&M University, 2009.

Pereira, M., "Towards Predictive Scale-Resolving Simulations of Turbulent External Flows," Phd thesis, Instituto Superior Técnico, Lisbon, 2018.

Razi, P., "Partially-averaged Navier-Stokes (PANS) Method for Turbulence Simulations: Near-wall Modeling and Smooth-surface Separation Computations," Phd thesis, Texas A\&M University, 2017.

Spalart, P. R., Jou, W. H., Strelets, M. K., and Allmaras, S. R., "Comments on the feasibility of LES for wings and on a hybrid RANS/LES approach," Advances in DNS/LES, 1(JANUARY):4-8, 1997.

Visonneau, M., Deng, G. B., Guilmineau, E., Queutey, P., and Wackers, J., "Local and Global Assessment of the Flow around the Japan Bulk Carrier with and without Energy Saving Devices at Model and Full Scale," 31st Symposium on Naval Hydrodynamics, number September, 2016.

Weller, H. G., Tabor, G., Jasak, H., and Fureby, C., "A Tensorial Approach to $\{\mathrm{CFD}\}$ using Object Oriented Techniques," Comput. Phys., 12:620, 1997.

Wilcox, D. C., "Turbulence Modeling for CFD (Second Edition),” 1998 ISBN 9781928729082. 